\title{
ADHESION MEASURES OF ELASTO-PLASTIC THIN FILM VIA BUCKLE-DRIVEN DELAMINATION
}

\author{
Yu Shouwen and Li Qunyang \\ Department of Engineering Mechanics, Tsinghua University, Beijing 100084, China \\ Yusw@mail.tsinghua.edu.cn
}

\begin{abstract}
Indentation test is becoming increasingly used to quantitatively assess the thin film interfacial adhesion for its simplicity and ability to mechanically probe the smallest of solids. The conventional technique is based on the analysis of Marshall and Evans which is a combination of Linear Elastic Fracture Mechanics (LEFM) and simplified post-buckling theory. In this paper a full post-buckling response of elasto-plastic thin film is investigated by FEM calculation; the contributions of double-buckling to the indentation test is discussed. The results show that double-buckling needs more energy than single-buckling case thus lead to a greater value of strain energy release rate
\end{abstract}

\section{Key Words}

Indentation, interfacial strength, thin film, delamination, double-buckling

\section{Introduction}

Thin films have a wide range of applications in microelectronics and magnetic recording industries. Because of the importance of thin film adhesion, it is not surprising that there are more than 200 different methods ${ }^{[1]}$ to measuring interfacial adhesion at present, suggesting them to be material, geometry and even industry specific. This indentation technique is mainly based on the pioneer works of Marshall \& Evans ${ }^{[7]}$ and Evans \& Hutchinson ${ }^{[8]}$ which gave the theoretical analysis for the conical indentation-induced thin film delamination.

Consider an indentation-induced interface crack in a residually stressed film, shown in Fig.1. The film has a thickness $t$ on a semi-infinite substrate, loaded by a hard angular indenter which leaves a permanent impression, and the residual stress is assumed to be $\sigma_{R}$. The strain energy release rate is obtained as follows by considering a few hypothetical operations $^{[7,8]}$

$$
G=t(1-v)\left\{(1-\alpha) \sigma_{R}^{2}+\sigma_{0}^{2}\left[(1+v) / 2-(1-\alpha)\left(1-\sigma_{c} / \sigma_{0}\right)^{2}\right]\right\} / E
$$

where $\alpha=1$ for $\sigma_{0}+\sigma_{R}<\sigma_{c}$ (no buckling) or $\alpha=(1+1.207(1+v))^{-1}$ for $\sigma_{0}+\sigma_{R}>\sigma_{c}$ (buckling). After measuring the strain energy release rate $G$, the interfacial adhesion between the thin film and substrate can be calculated, which needs the knowledge of the fracture interface and the phase angle to interpret the results correctly ${ }^{[1]}$.

In many indentation tests, if the indenter is driven deep enough, so that the crack reaches its critical buckling length, the film often double buckles during indentation, shown in Fig.2 


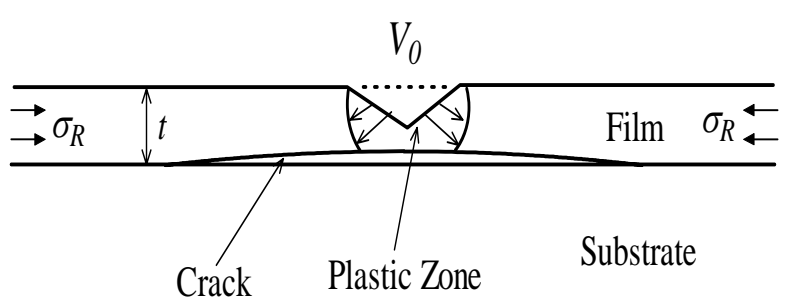

Fig.1 Schematic of indentation-induced delamination at the interface of a thin film and substrate

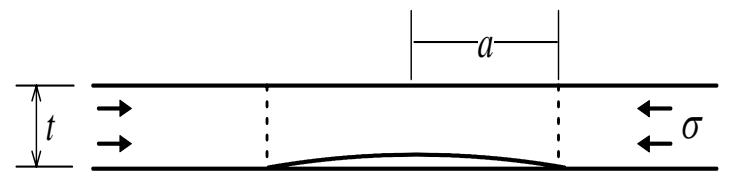

(a)

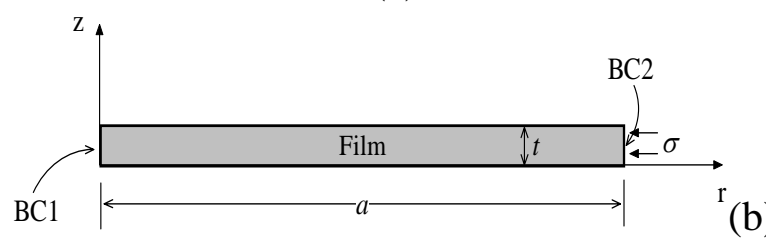

Fig. 3(a) Circular delamination at afilm/substrate interface; (b) Model for FEM calcutlation (a)

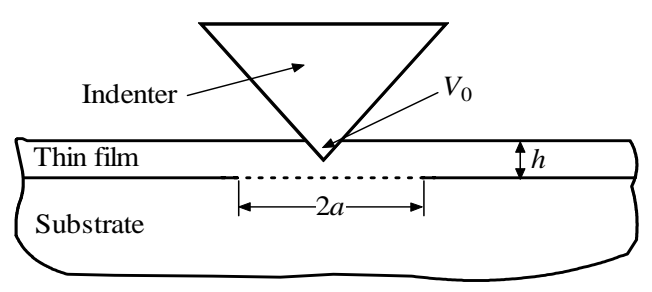

(b)

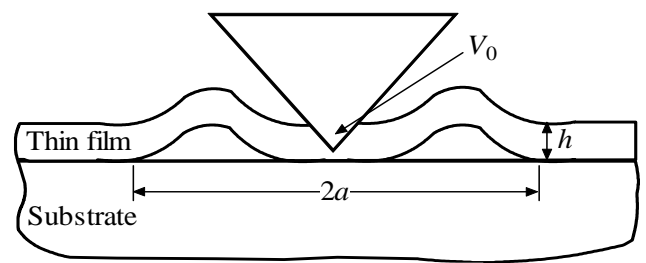

(c)

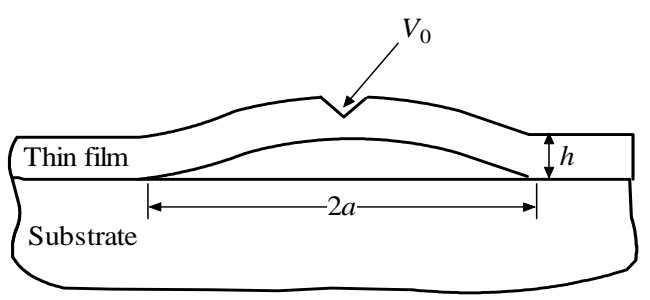

Fig.2 (a) No buckling during indentation;

(b) double-buckling during indentation;

(c)single-buckling after the indenter tip removal, see ${ }^{[6,9]}$

with the plastic indentation volume $V_{0}$. But as what is mentioned above, the full analysis of double-buckling has not yet been done, which is essential for the indentation technique. Experiment of Kriese et al. ${ }^{[9]}$ found that during indentation the interfacial fracture toughness was reproducibility high for shallow indents, $8-10 \mathrm{~J} / \mathrm{m}^{2}$, but dropped to a fairly steady $0.7-1.2 \mathrm{~J} / \mathrm{m}^{2}$ for deeper indents. It is hard to explain these experimental data via conventional analysis. As we know, the plasticity of materials will greatly affect the buckling process; can it be a reason for this phenomenon.

In the paper, we will emphasize on some aspects of thin film bucking and their influences to the indentation test. Based on FEM code ABAUQS, the full post-buckling responses of thin films are obtained. Both the double-buckling and material plasticity are taken into consideration and their contributions are discussed.

\section{Large deformation buckling and double-buckling for thin film delamination}

\subsection{Model for FEM analysis}

The calculation is done by the commercial FE-code ABAQUS on a PC workstation. A circular delamination at a film/substrate interface is considered, as shown in Fig.3(a), with a uniform biaxial compression $\sigma$ exiting in the film. For this axisymmetric problem, only one cross-section of the buckled film (i.e. the buckled film disc) is modeled, shown in Fig.3(b).

The film disc has a thickness $t$ and a radius $a$, and is subject to uniform biaxial compression $\sigma$ on the perimeter (BC2). Boundary conditions are applied, such that the 
perimeter (BC2) is always fixed in $r$ direction while the film center (BC1) is fixed in $r$ direction for single-buckling case but fixed in both $r$ and $z$ direction for double-buckling case.

\subsection{FEM results of full single-buckling response}

The procedure of 'Eigenvalue Buckling Prediction' is used to obtain the critical buckle stress. For various values of $t / a$, using the non-dimensional stress $\bar{\sigma}_{c}=12\left(1-v^{2}\right) \sigma_{c} / E$, the relevant critical load for the thin film is shown in Fig.4

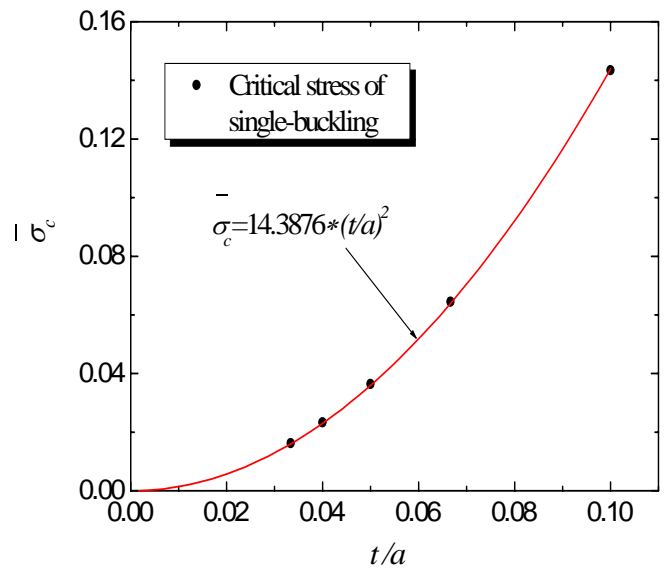

Fig. 4 Critical buckle stress for film discs with different radius (single-buckling)

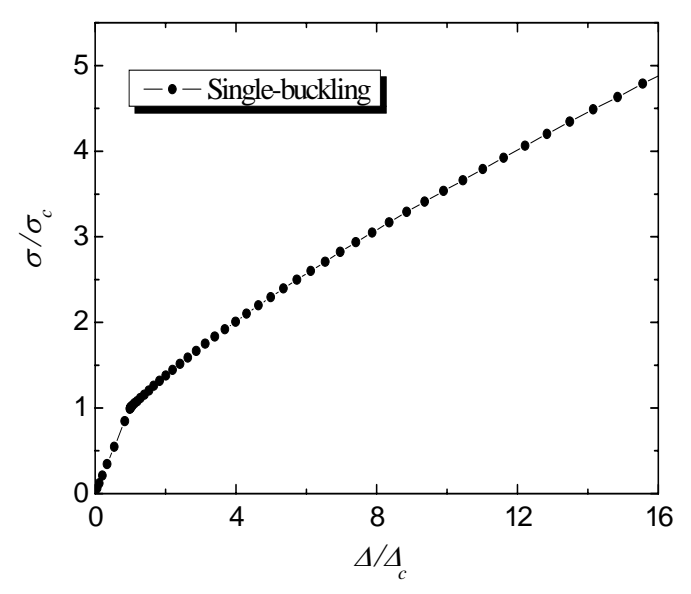

Fig.5 Plot of edge stress $\sigma$ as a function of inward displacement $\Delta$ ( $\sigma_{c}$ and $\Delta_{c}$ are the critical stress and displacement for singlebuckling)

Fitting the above data, we get that the critical stress follows the form

$$
\sigma_{c}=\frac{k E}{12\left(1-v^{2}\right)} \cdot\left(\frac{t}{a}\right)^{2}
$$

with $k=14.3876$, which coincides the theoretical Euler buckling stress $k=14.68$ very well.

To calculate the film response when $\sigma>\sigma_{c}$, RIKS procedure is used to perform postbuckling analyses:

For the case of $t / a=1 / 20$, the edge stress $\sigma$ as a function of displacement $\Delta$ for this film disc is plotted in Fig.5.

Fig.5 gives the full post-buckling response of the elastic thin film, from which we can clearly observe the whole process of buckling and the stiffness of the film (i.e. the slope of the curve) is greatly reduced after the critical point of buckling. The initial slope $\alpha$ of the post-buckled load-displacement curve brunches are drawn in Fig.6 for materials with different Poisson's ratio $v$. 


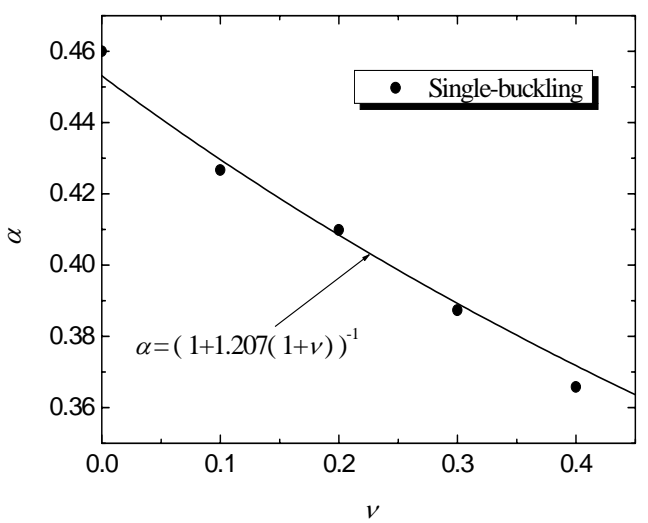

Fig.6 The initial slope $\alpha$ of buckled brunches verse film Poisson's ratio $v$ for single-buckling

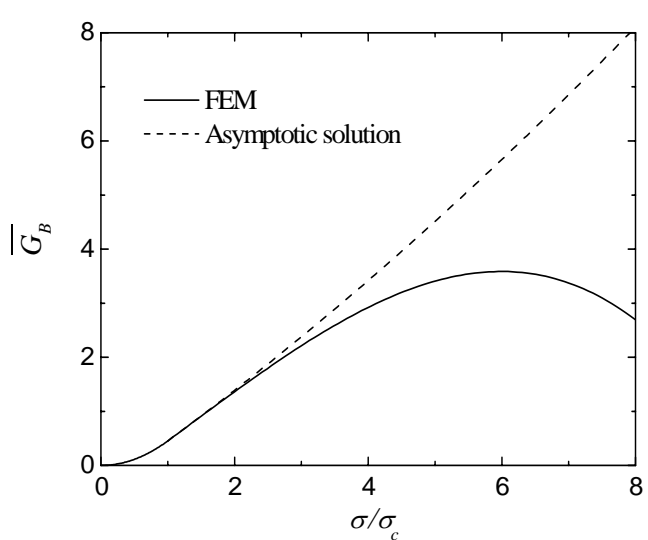

Fig.7 Energy release rate of FEM-based and asymptotic-solution-based results

$$
\left(\bar{G}_{B}=\sigma_{c}^{2} t / E\right)
$$

The FEM results of the initial slope well fit the theoretical prediction, which is provided by Evans \& Hutchinson ${ }^{[8]}$ and assumed to be $\alpha=(1+1.207(1+v))^{-1}$. But from Fig.5, it can be seen that the slope of the post-buckled brunch goes down as the increase of inward displacement. To consider the deviation from the initial slope value, we assume the slope $\alpha$ has the following form

$$
\alpha=\alpha_{0}-\beta \frac{\Delta}{\Delta_{c}}
$$

where $\alpha_{0}$ is the initial slop value and $\beta$ is fitted from the FEM data to be around 0.01973 for different values of $v$. Thus the equationFel! Hittar inte referenskälla. is modified as

$$
G=\frac{t(1-v)}{E}\left\{(1-\alpha) \sigma_{R}^{2}-\frac{\beta}{2}\left(\sigma_{0}+\sigma_{c}-\sigma_{R}\right)^{2}+\sigma_{0}^{2}\left[\frac{1+v}{2}-(1-\alpha+\beta)\left(1-\frac{\sigma_{c}}{\sigma_{0}}\right)^{2}\right]\right\}
$$

where $\alpha$ and $\beta$ are determined by equation (2).

In the initial study we assume there is no pre-residual stress in the thin film, and the comparison of strain energy release rate between the result of equationFel! Hittar inte referenskälla. and equation(3) is shown in Fig.7.

The comparison of Fig.7 shows that the relative error of the asymptotic solution is within $10 \%$ when $\sigma<3 \sigma_{c}$, but the results deviate a lot when large deformation is taken place. For general indentation test of relatively low value of $\sigma / \sigma_{c}$, the asymptotic solution based results are satisfactory for engineering applications.

\subsection{FEM results of double-buckling response}

The following will focus on the double-buckling responses, which is shown in Fig.2(b).

First, we also calculate the critical stress $\sigma_{c}^{\prime}$ for different values of $t / a$ when the films double-buckled, plot is shown in Fig.8 with $\bar{\sigma}_{c}=12\left(1-v^{2}\right) \sigma_{c}^{\prime} / E$. 


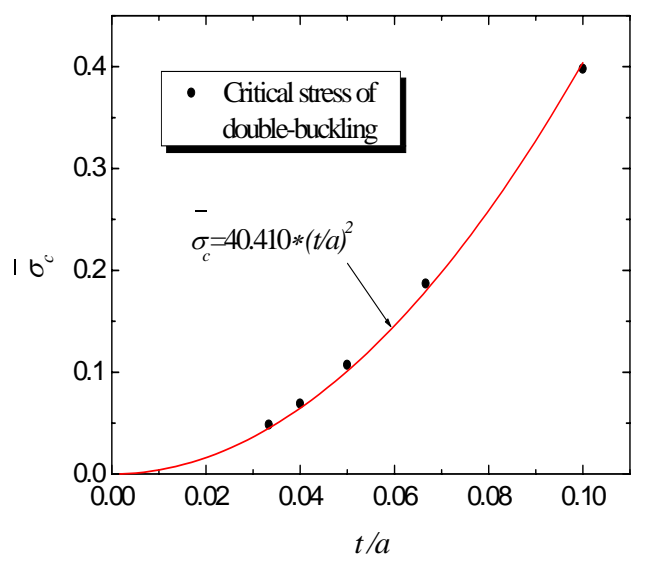

Fig.8 Critical buckle stress for film discs with different radius (double-buckling)

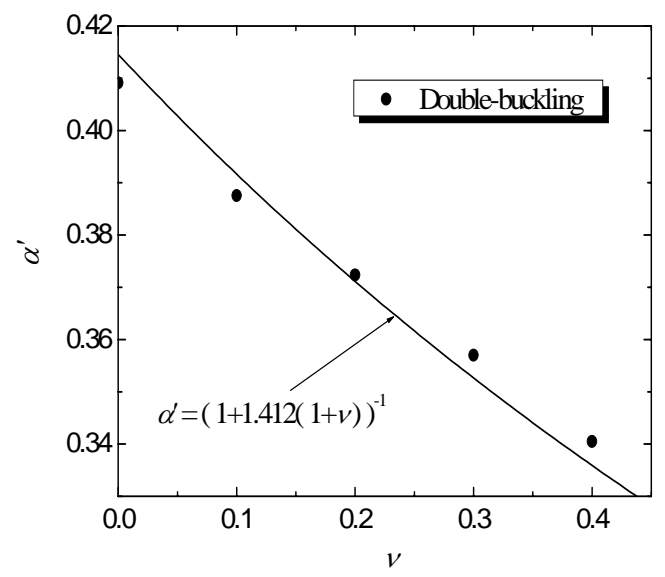

Fig.10 The initial slope $\alpha^{\prime}$ of buckled brunches verse film Poisson's ratio $v$ for double-buckling cases

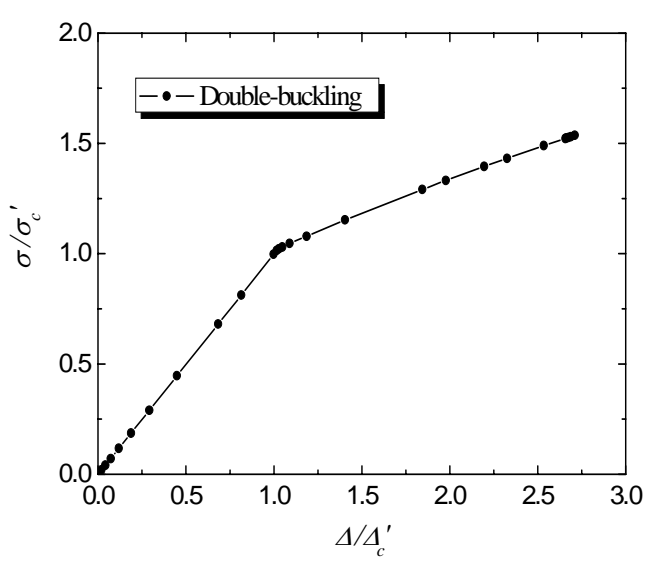

Fig.9 Plot of edge stress $\sigma$ as a function of inward displacement $\Delta .\left(\sigma_{c}^{\prime}\right.$ and $\Delta_{c}^{\prime}$ are the critical stress and displacement for doublebuckling)

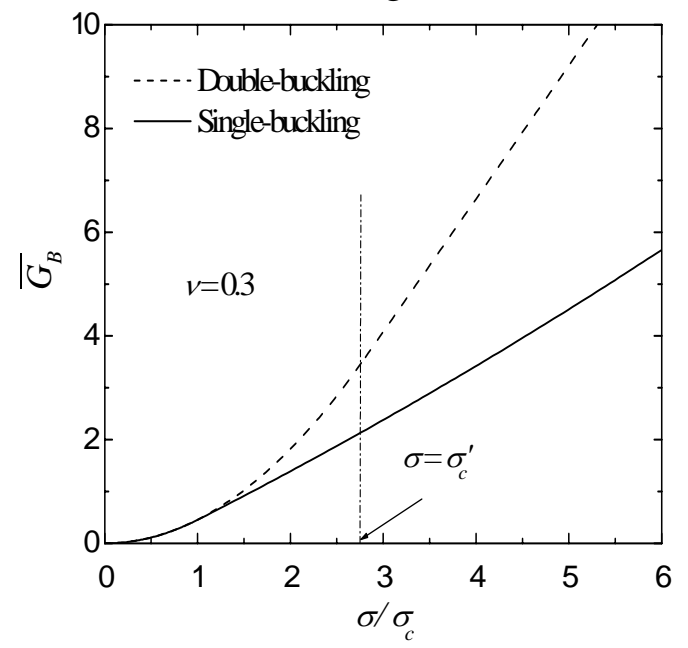

Fig.11 Comparison of energy release rate for the two buckling types

Fitting the FEM data, we can get the critical stress for double-buckling

$$
\sigma_{c}^{\prime}=\frac{k^{\prime} E}{12\left(1-v^{2}\right)} \cdot\left(\frac{t}{a}\right)^{2}
$$

where $k^{\prime}=40.410$, very close to the Euler theoretical critical value of $k^{\prime}=42.67$.

For the case of double-buckling, the full post-buckling response of the edge stress $\sigma$ as a function of displacement $\Delta$ for this film disc is plotted in Fig.9.

While the initial slope $\alpha^{\prime}$ of the post-buckled load-displacement curve brunches are drawn in Fig. 10 for materials with different Poisson's ratio $v$.

The relationship between $\alpha^{\prime}$ and $v$ can also be fitted from the FEM results and expressed as

$$
\alpha^{\prime}=(1+1.412(1+v))^{-1}
$$


from equation(5) we can see that though the critical stress value of double-buckling is much larger than that of single-buckling.

Considering the difference of the critical buckled stress and its initial slope value of postbuckled brunches, the corresponding strain energy release rate of double-buckling will be larger. The comparison of the strain energy release rate for the two buckling types is shown in Fig.11, with the non-dimensional parameter $\bar{G}_{B}=\sigma_{c}^{2} t / E$ and $v=0.3$.

In Fig.11, it can be seen that the double-buckling case has a much larger strain energy release rate than single-buckling case, so we must identify the buckling type in order to interpret the results correctly.

\section{Conclusion}

An investigation on the post-buckling of thin film is carried out by FEM calculation. Some of the important factors, which are often omitted before, such as the double-buckling phenomenon and material plasticity, are discussed in this paper.

The results show that for the case of $\sigma<3 \sigma_{c}$, the asymptotical solution is satisfactory with a relative error less than $10 \%$. For the double-buckling cases, the critical stress and initial slope parameter are obtained, and the comparison of the energy release rate with the singlebuckling case shows that the different is obvious and need to be treated properly.

The plasticity has significant influence on the post-buckling responses and should be considered in interpreting the indentation test results. The greater the value of $\sigma_{c} / \sigma_{y}$, the more contributions of the plasticity. The abnormal experimental results of Keries ${ }^{[9]}$ can be explained by this calculation. We can say that if the conventional method of indentation test is considered, the deeper indent the more precise of the results.

\section{Acknowledgement}

This project is supported by NSFC (10172050, 10121202 ) and SRFDP 3064,Key grant proj-of Chinese MoE-No0306.

\section{References}

1. Volinsky A A, Moody N R, Gerberich W W. Acta Materialia. vol. 50, 441-466, 2002

2. Jindal P C, Quinto D T, Wolfe G J. Thin Solid Films. vol. 154, 361-375, 1987

3. Rickerby D S. Surf. Coat. Tech. vol. 36, 541-559, 1988

4. Steinmann P A, Hintermann H E. J. Vac. Sci. Technol. A. vol. 7, 2267-2272,1989

5. McCabe A R, Jones A M, Bull S J. Diamond and Related Technol. vol. 3, 205-209,1994

6. Gerberich W W, Kramer D E, et al. Acta. Mater. vol. 47(15), 4115-4123, 1999

7. Marshall D B, Evans A G. J. Appl. Phys. vol. 56(10), 2632-2638, 1984

8. Evans A G, Hutchinson J W. Int. J. Solids Structures. vol. 20(5), 455-466, 1984

9. Krese M D, Boismier D A, Moody N R, Gerberich W W. Engineering Fracture Mechanics. vol. 61, 1-20, 1998 